\title{
Exhaled breath condensate as an alternative sample for drug monitoring
}

\author{
Maryam Khoubnasabjafari ${ }^{1}$, Elaheh Rahimpour ${ }^{2}$ \& Abolghasem Jouyban ${ }^{*}, 3$ \\ ${ }^{1}$ Tuberculosis \& Lung Diseases Research Center, Tabriz University of Medical Sciences, Tabriz, Iran \\ ${ }^{2}$ Drug Applied Research Center, Tabriz University of Medical Sciences, Tabriz, Iran \\ ${ }^{3}$ Pharmaceutical Analysis Research Center \& Faculty of Pharmacy, Tabriz University of Medical Sciences, Tabriz, Iran \\ * Author for correspondence: Fax: +98 413336 3231; ajouyban@hotmail.com
}

\begin{abstract}
"Concerning recent promising findings in the analysis of drugs in EBC and the highly increased demands for therapeutic drug monitoring and related biomedical analyses, finding an appropriate noninvasive sample is a priority."
\end{abstract}

First draft submitted: 16 September 2017; Accepted for publication: 2 November 2017; Published online: 13 December 2017

Keywords: biological samples $\bullet$ biomarkers $\bullet$ drugs $\bullet$ exhaled breath condensate

Blood, urine, saliva, cerebrospinal fluid, feces, tear, sweat, nail and hair are all commonly used samples in bioanalysis. Such biological samples can be used for many different purposes, including the early detection of disease, the follow-up on a cause of death in forensic science, drug dose adjustment in modern pharmacotherapy and for measuring enzyme activity in order to obtain phenotypic information for personalized medicine. Each of these biological samples has a number of advantages and disadvantages; however, serum and plasma are currently the most frequently used sample in clinical analysis. The levels of drugs in the blood, or its derived fluids, reflect the systemic levels of the analytes, and so these types of samples are widely accepted in the biomedical field. However, these samples possess a number of disadvantages, including: invasive sampling, the necessity for a skilled person to collect the sample, a very high matrix effect, patient-to-patient variability and a low compliance of the patients and/or sample donors.

Exhaled breath (EB)/exhaled breath condensate (EBC) could be considered as a possible alternative sample type for drug monitoring purposes and also for the early detection/follow-up of disease through the monitoring appropriate biomarker levels [1] or the response to pharmacotherapy [2]. This editorial focuses on the advantages and disadvantages, as well as the recent findings, of the analysis of drugs in EBC. A number of important issues that should be resolved before further investigations on EBC will also be discussed.

\section{Characteristics of exhaled breath condensate}

The main advantages of using EBC include:

- A simpler matrix (low-matrix effect) when compared with other biological fluids;

- A noninvasive nature and therefore greater compliance from patients during EBC collection;

- No need for a skilled person to collect EBC;

- Sampling could be repeated as frequently as required;

- Direct sample injection into the analytical instruments is possible;

- A greater possibility for chiral separation of enantioselective analytes.

The main disadvantages of using EBC include:

- A very low concentration of drugs/biomarkers due to dilution by the water vapor of exhaled air;

- Different units are used to report the analytical data meaning that the data cannot easily be directly compared;

- Possible contamination from the collection area (inhaled air) and also the saliva; 
- A requirement for expensive and more sensitive analytical procedure to monitor low concentrations of drugs/biomarkers present in EBC;

- EBC is mostly studied for the lung diseases and so further investigations are required to confirm potential applications of EBC for analyzing systemic changes of drug/biomarker concentrations;

- Adsorption of analytes to the surface of the collection setup;

- Low reproducibility for EBC samples collected using different setups;

- Lack of any standardization method for the final validation of the data;

- Variability of analyte concentrations due to collection area conditions (i.e., temperature, humidity, etc.);

- Unavailability of an accepted dilution marker for EBC samples;

- Patient-to-patient variability in EBC volume and the effect of lung function on the collected sample volume.

From an analytical point of view, the main advantages of EBC are its very low matrix effect due to its simple matrix and its ability to be directly injected into an analytical system [3]. This latter point, however, is associated with its main disadvantage: a very low concentration of drugs requiring highly sophisticated analytical techniques.

Over the last decade, chromatographic methods have become the technique of choice for the trace analysis of various compounds contained in complex biological matrices. In order to achieve a relatively low detection limit in EBC samples, it is often necessary to incorporate preconcentration steps (e.g., solid-phase extraction, lyophilization and liquid-liquid microextraction) prior to analysis. For example, Göen $e t$ al. used a clean-up and preconcentration step for 3-nitrotyrosine measurement in EBC using a $\mathrm{C}_{18}$ cartridge, before LC-MS/MS analysis [4]. In addition, Khoubnasabjafari et al. used two sample preconcentration/preparation procedures (i.e., dispersive liquid-liquid microextraction and ultrasonic liquid-liquid microextraction) before HPLC-UV analysis for determination of methadone in the EBC samples [5]. Karyakina $e t$ al. reported a flow-injection analysis electrochemical system coupled with an ion-exchange preconcentration column (filled with Strata SAX sorbent-silica gel with quaternary ammonium substituents, Phenomenex, CA, USA) for the lactate sensing in EBC of patients with lung diseases [6]. Beck et al. also used a solid phase extraction cartridge (Varian, CA, USA) for the extraction and preconcentration of methadone from EBC prior to LC-MS/MS determination [7].

\section{Drug levels in exhaled breath/exhaled breath condensate}

A number of drugs have been analyzed in EB/EBC in various studies [8-19]. However, there are two problems when comparing these reported levels of drugs in EBC: there are very wide variations in the reported levels (e.g., 4.8$29,800 \mathrm{pg} / \mathrm{min}$ for methadone [12]) and the different units (i.e., $\mathrm{pg} /$ filter, $\mathrm{pg} / \mathrm{min}, \mathrm{mg} / \mathrm{l}$ and parts per million by volume) used to express the EBC levels of the analyte which cannot easily be converted for comparison with each other. Variations in analyte concentrations can be partially controlled using normalization by the sum of sodium and potassium concentrations [20] or by measuring the conductivity [21] of the collected EBC samples. Regarding the different units used, a standard and validated collection device and an approved collection procedure should be agreed upon by an expert panel, so that a harmonized unit can be proposed for reporting the levels of analytes in EBC. Concerning further improvements in the sample collection procedure, one deep EB should provide a sufficient volume of EBC sample for the determination of trace levels of analytes. However, this is only if one could combine the progresses made in microfluidic, electronic noses, paper-based point-of-care tests, optical/electrochemical sensors, lab-on-a-chip or other advanced methods with a miniaturized EBC collection device.

The very large surface area of the capillary blood vessels in the lung enables the establishment of an equilibrium between the plasma and lung lining fluid for various analytes, and the analytes can then penetrate from the lung lining fluid into the EBC. This provides the possibility for the follow-up of systemic levels of analytes using EBC (as an alternative biological sample to blood) and their correlation with clinical findings. It is expected that all drugs present in the blood should also be present in the lung lining fluid, and therefore consequently in the EBC. There is the evidence of good plasma-EBC correlation, for example for propofol [16]. These sorts of correlation studies should be further investigated using drugs with better pharmacokinetic properties, and the more technical points of collecting and analyzing EBC samples should be considered. Such technical considerations include: standardization with respect to collection temperature, the materials used in the inner surface of the collection setup, flow design, as well as other parameters such as ventilatory patterns, tidal volumes, breathing frequencies and exhaled particles. 


\section{Review articles focused on exhaled breath condensate analysis}

Horvath et al. summarized the 'American Thoracic Society/European Respiratory Society Task Force on EBC' comments and technical recommendations on EBC collection and assay methods with an invaluable review [22]. The group focused on EBC analysis in lung disease patients only; however, it is believed that EBC samples could be used for monitoring analytes related to systemic diseases, as well as orally administered drugs, as shown in the recent works $[18,19]$. Horvath et al. provided the following general recommendations for EBC collection: "Collections should occur during tidal breathing using a noseclip and a saliva trap, with a defined cooling temperature and collection time; surfaces contacting the EBC should be inert to the compounds of interest in a given study; inclusion of expiratory flow resistance or filters is not required" [22]. All technical points and recommendations should be considered in the research using EBC to provide standard and comparable results.

Kuban and Foret reviewed the historical background and various available collection devices for EBC [23]. They highlighted EBC as a novel biological sample with potential to become one of the simplest diagnostic fluids due to its complete noninvasiveness, easy and inexpensive collection and very simple matrix composition. They summarized the recent advances in the analytical methods and the potential of EBC in various biomedical applications. Beck et al. reviewed the recent works on more selective collection procedures for exhaled particles and discussed the potential of MS for the analysis of nonvolatile biomarkers present in EBC [10]. Here, it was concluded that despite the unique sensitivity and selectivity of MS, it is not a cost-effective method for the routine analyses of $\mathrm{EB}$, especially in large studies. Amann $e t$ al. also described the breath analysis as a young field of research with great clinical potential [24]. Here, the developments in the analysis of volatile compounds (e.g., endogenously produced volatiles, metabolites of ingested precursors, metabolites produced by bacteria in the gut or the airways or volatiles appearing after environmental exposure in the breath using GC-MS) and the possibility of real time analysis were reviewed. A number of analyses on EBC have been approved by the US FDA, including determination of carbon dioxide in the $\mathrm{EBC}$ of the patients with neonatal jaundice, measurement of nitric oxide in asthmatic patients and monitoring of hydrogen and methane levels in the EBC samples of patients with gastrointestinal diseases [24].

\section{Conclusion \& future perspective}

Concerning recent promising findings in the analysis of drugs in EBC and the highly increased demands for therapeutic drug monitoring and related biomedical analyses, finding an appropriate noninvasive sample is a priority. A number of associated problems with EBC could be resolved easily. For example, any sample contamination could be removed by using a saliva filter and background interferents (or contamination from the air) could be removed by analysis of inhaled air or air from the collection area then subtracting the analytes levels found in the EBC from those of the inhaled air. Concerning recent advances in the analytical area, the cost of analysis and the demands in clinical practice for cheap, simple and straightforward analytical methods should be balanced when making the final decision on the applicability of monitoring a drug in EBC. Therefore, although EBC holds great promise for applications in therapeutic drug monitoring studies, as discussed above, further investigations should be carried out before it can be used as a suitable replacement for blood samples in the bioanalytical research.

Financial \& competing interests disclosure

Patent: A Jouyban, M Khoubnasabjafari, K Ansarin and V Jouyban-Gharamaleki. Breath sampling setup. Iranian Patent, 81363 , 2013. The authors have no other relevant affiliations or financial involvement with any organization or entity with a financial interest in or financial conflict with the subject matter or materials discussed in the manuscript apart from those disclosed.

RD Vooght-Johnson (Freelance Scientific Writer and Editor [Royston, UK]) proofread the draft of the manuscript and the language editing payment was made by Tabriz University of Medical Sciences.

\section{References}

1. Krilaviciute A, Heiss JA, Leja M et al. Detection of cancer through exhaled breath: a systematic review. Oncotarget 6(36), 38643-38657 (2015).

2. Nardi-Agmon I, Abud-Hawa M, Liran O et al. Exhaled breath analysis for monitoring response to treatment in advanced lung cancer. J. Thoracic Oncol. 11(6), 827-837 (2016).

3. Hamidi S, Khoubnasabjafari M, Ansarin K et al. Direct analysis of methadone in exhaled breath condensate by capillary zone electrophoresis. Curr. Pharm. Anal. 12(13), 137-145 (2016).

4. Göen T, Müller-Lux A, Dewes P et al. Sensitive and accurate analyses of free 3-nitrotyrosine in exhaled breath condensate by LC-MS/MS. J. Chromatogr. B 826(1), 261-266 (2005). 
5. Khoubnasabjafari M, Ansarin K, Jouyban-Gharamaleki $\mathrm{V}$ et al. Extraction and analysis of methadone in exhaled breath condensate using a validated LC-UV method. J. Pharm. Sci. Pharmacol. 18(2), 207-219 (2015).

6. Karyakina EE, Lukhnovich AV, Yashina EI et al. Electrochemical biosensor powered by preconcentration: improved sensitivity and selectivity toward lactate. Electroanalysis 28, 2389-2393 (2016).

7. Beck O, Sandqvist S, Eriksen P et al. Determination of methadone in exhaled breath condensate by liquid chromatography-tandem mass spectrometry. J. Anal. Toxicol. 35(3), 129-133 (2011).

8. Manolis A, McBurney LJ, Bobbie BA. The detection of delta 9-tetrahydrocannabinol in the breath of human subjects. Clin. Biochem. 6(4), 229-233 (1983).

9. Beck O, Sandqvist S, Dubbelboer I, Franck J. Detection of $\Delta^{9}$-tetrahydrocannabinol in exhaled breath collected from cannabis users. J. Anal. Toxicol. 35(8), 541-544 (2011).

10. Beck O, Onlin A, Mirgorodskaya E. Potential of mass spectrometry in developing clinical laboratory biomarkers of nonvolatiles in exhaled breath. Clin. Chem. 62(1), 84-91 (2016).

11. Beck O, Leine K, Palmskog G, Franck J. Amphetamines detected in exhaled breath from drug addicts: a new possible method for drugs-of-abuse testing. J. Anal. Toxicol. 34, 233-237 (2010).

12. Beck O, Stephanson N, Sandqvist S, Franck J. Detection of drugs of abuse in exhaled breath from users following recovery from intoxication. J. Anal. Toxicol. 36(9), 638-646 (2012).

13. Beck O, Sandqvist S, Böttcher M et al. Study on the sampling of methadone from exhaled breath. J. Anal. Toxicol. 35(5), 257-263 (2011).

14. Khoubnasabjafari M, Ansarin K, Jouyban-Gharamaleki V et al. Methadone concentrations in exhaled breath condensate, serum and urine of patients under maintenance treatment. Iran J. Pharm. Res. 16(4), 1621-1630 (2017).

15. Meyer MR, Rosenborg S, Stenberg M, Beck O. First report on the pharmacokinetics of tramadol and $O$-desmethyltramadol in exhaled breath compared to plasma and oral fluid after a single oral dose. Biochem. Pharmacol. 98(3), 502-510 (2015).

16. Miekisch W, Fuchs P, Kamysek S et al. Assessment of propofol concentrations in human breath and blood by means of HS-SPME-GC-MS. Clin. Chim. Acta 395(1), 32-37 (2008).

17. Ghimenti S, Di Francesco F, Onor M et al. Postoperative elimination of sevoflurane anesthetic and hexafluoroisopropanol metabolite in exhaled breath: pharmacokinetic models for assessing liver function. J. Breath. Res. 7(3), 036001 (2013).

18. Jouyban A, Samadi A, Khoubnasabjafari M et al. Amidosulfonic acid capped silver nanoparticles as a new spectrophotometric probe for rapid quantification of lamotrigine in exhaled breath condensate. Microchim. Acta 184, 2991-2998 (2017).

19. Hamidi S, Amini M, Khoubnasabjafari M et al. LC-MS determination of propranolol in exhaled breath condensate. Pharm. Sci. doi:10.15171/PS.2017.39 (2017) (Epub ahead of print).

20. Effros RM, Hoagland KW, Bosbous M et al. Dilution of respiratory solutes in exhaled condensates. Am. J. Respir. Crit. Care Med. 165(5), 663-669 (2002).

21. Effros RM, Biller J, Foss B et al. A simple method for estimating respiratory solute dilution in exhaled breath condensates. Am. J. Respir. Crit. Care Med. 168(12), 1500-1505 (2003).

22. Horvath I, Hunt J, Barnes PJ et al. Exhaled breath condensates: methodological recommendations and unresolved questions. Eur. Respir. J. 26, 523-548 (2005).

23. Kubáň P, Foret F. Exhaled breath condensate: determination of nonvolatile compounds and their potential for clinical diagnosis and monitoring: a review. Anal. Chem. Acta 805, 1-18 (2013).

24. Amann A, Miekisch W, Schubert J et al. Analysis of exhaled breath for disease detection. Annu. Rev. Anal. Chem. 7, 455-482 (2014) 